\title{
S-1-Induced Lacrimal Drainage Obstruction and Its Association with Ingredients/Metabolites of S-1 in Tears and Plasma: A Prospective Multi-institutional Study
}

\author{
Namju Kim, MD ${ }^{1}$ \\ Jin Won Kim, MD² \\ Je-Hyun Baek, PhD ${ }^{3}$ \\ Jin-Soo Kim, MD ${ }^{4}$ \\ Ho-Kyung Choung, $\mathrm{MD}^{5}$ \\ Tae-Yong Kim, $\mathrm{MD}^{6}$ \\ Kyung-Hun Lee, $\mathrm{MD}^{6}$ \\ Yung-Jue Bang, $\mathrm{MD}^{6}$ \\ Sang In Khwarg, $\mathrm{MD}^{7}$ \\ Sang-Hoon Ahn, MD \\ Do Joong Park, MD ${ }^{8}$ \\ Hyung-Ho Kim, MD \\ Jae-Yong Chung, MD9 \\ Soyeon Ahn, PhD ${ }^{10}$ \\ Keun-Wook Lee, MD2
}

*A list author's affiliations appears at the end of the paper.

\author{
Correspondence: Keun-Wook Lee, MD \\ Department of Internal Medicine, Seoul National \\ University Bundang Hospital, Seoul National \\ University College of Medicine, 166 Gumi-ro, \\ Bundang-gu, Seongnam 16320, Korea \\ Tel: 82-31-787-7037 \\ Fax: 82-31-787-4098 \\ E-mail: hmodoctor@snubh.org \\ Received December 2, 2016 \\ Accepted February 12, 2017 \\ Published Online February 27, 2017
}

Key words

Lacrimal drainage obstruction, S-1, Fluorouracil, Tears,

Stomach neoplasms

\section{Purpose}

This prospective study was conducted to determine the incidence of lacrimal drainage obstruction (LDO) during S-1 chemotherapy and evaluate the association between the development of LDO and the concentrations of ingredients/metabolites of S- 1 in tears and plasma.

\section{Materials and Methods}

A total of 145 patients with gastric cancer who received adjuvant S-1 therapy were enrolled. Ophthalmologic examinations were performed regularly during S-1 chemotherapy. Concentrations of tegafur, 5-chloro-2,4-dihydroxypyridine (CDHP), and 5-fluorouracil at steady-state trough level were measured in both tears and plasma.

\section{Results}

Fifty-three patients (37\%) developed LDO. The median time to the onset of LDO was 10.9 weeks, and LDO developed most frequently in the nasolacrimal duct. Univariable analyses revealed that an older age ( $\geq 70$ years), creatinine clearance rate $(\mathrm{Ccr})<80 \mathrm{~mL} / \mathrm{min}, 5$-fluorouracil concentration in plasma $\geq 22.3 \mathrm{ng} / \mathrm{mL}$ (median), CDHP concentration in plasma $\geq 42.0 \mathrm{ng} / \mathrm{mL}$ (median), and tegafur concentration in tears $\geq 479.2 \mathrm{ng} / \mathrm{mL}$ (median) were related to increased development of LDO. Multivariable analysis indicated that a high plasma 5-fluorouracil concentration was predictive of increased development of LDO (hazard ratio, 2.02; $p=0.040$ ), along with older age and decreased Ccr. Patients with LDO also developed S-1-related non-hematologic toxicity more frequently than those without LDO $(p=0.016)$.

\section{Conclusion}

LDO is a frequent adverse event during S-1 chemotherapy. An older age, decreased Ccr, and high plasma 5-fluorouracil concentration were found to be independent risk factors for LDO. The high incidence of LDO warrants regular ophthalmologic examination and early intervention in patients receiving S-1 therapy. 


\section{Introduction}

S-1 is an oral antineoplastic agent widely used for the treatment of gastric cancer and other gastrointestinal cancers. S-1 consists of tegafur (5-fluorouracil prodrug), 5-chloro-2, 4-dihydroxypyridine (CDHP), and potassium oxonate [1]. Lacrimal drainage obstruction (LDO) is an interesting adverse effect of antineoplastic agents that is more commonly associated with S-1 [2-5]. We previously performed a large-scale retrospective study that revealed the incidence of LDO after S-1 treatment is not infrequent [2]. However, all studies conducted to date were retrospective in nature [2-5], and the development mechanisms of S-1-induced LDO have not yet been systematically investigated.

In this study, we investigated whether an ingredient (tegafur or CDHP) or active metabolite (5-fluorouracil) of S-1 directly influenced the development of S-1-induced LDO. To determine if LDO development is caused by a local effect (direct damage to the lacrimal drainage system by either 5-fluorouracil or an ingredient of S-1 in tears) or by one of the systemic adverse effects of $S-1$, we measured the concentrations of tegafur, CDHP, and 5-fluorouracil in both tears and plasma. To our knowledge, this is the first prospective study to investigate the incidence of S-1-induced LDO and explore the association between the LDO development and the concentrations of ingredients / metabolites of S-1 in tears and plasma.

\section{Materials and Methods}

\section{Patient population and adjuvant S-1 chemotherapy}

Patients with gastric or gastroesophageal junction adenocarcinoma, who underwent curative gastrectomy with D2 lymph node dissection and received adjuvant $S-1$ chemotherapy, were enrolled from three institutions between December 2010 and June 2013. Before the initiation of S-1 chemotherapy, the patients had adequate bone marrow, renal and hepatic functions, and fully recovered from gastrectomy. The planned duration of S-1 chemotherapy was 1 year. S- 1 was orally administered twice a day for 4 weeks, followed by a 2-week rest period (every 6 weeks). The dose of S-1 was determined as follows: $60 \mathrm{mg}$ for a body surface area (BSA) of $\geq 1.50 \mathrm{~m}^{2} ; 50 \mathrm{mg}$ for a BSA of $1.25-1.49 \mathrm{~m}^{2}$; and $40 \mathrm{mg}$ for a BSA of $<1.25 \mathrm{~m}^{2}$. The S-1 dose and schedule modification based on toxicity was described in detail previously $[2,6,7]$. Data describing S-1-related adverse events were collected until the completion of S-1 treatment. Adverse events were graded using National Cancer Institute Common Terminology Criteria for Adverse Events ver. 4.0. This study was approved by the Institutional Review Boards at the three institutions and followed the Declaration of Helsinki. Written informed consent was obtained from all patients.

\section{Ophthalmologic evaluation}

Comprehensive ophthalmic assessments were routinely performed until the completion of S-1 therapy (at 3 weeks and 3, 6, 9, and 12 months after the initiation of S-1) or until LDO development during S-1 therapy. Patients who had preexisting LDO at the baseline examination before the S-1 initiation were excluded. Ophthalmologic assessments consisted of visual acuity and intraocular pressure measurements; namely, slit-lamp examination to measure the tear meniscus height, a fluorescein dye disappearance test (FDDT) to estimate the physiological tear drainage function and a lacrimal irrigation test to identify the lacrimal drainage patency. The LDO was defined as partial or total obstruction of the lacrimal drainage system when all of the following conditions were met: high tear meniscus, delayed FDDT, and partial or total regurgitation on the lacrimal irrigation test. Once LDO was diagnosed, diagnostic lacrimal probing was routinely performed to locate the site of obstruction. Dacryocystography was recommended to the patients who developed LDO.

\section{Collection of tears and blood}

Tears and blood were obtained on the third week after initiation of S-1 chemotherapy, immediately before the patients took S-1, when S-1 was at a steady-state trough level. If samples could not be acquired at this time point, sample acquisition at another time point was allowed if the patient took S-1 for at least 7 consecutive days. The detailed methods for the collection of tears and blood are described in the Supplementary Methods.

\section{Measurement of tegafur, $\mathrm{CDHP}$, and 5-fluorouracil con- centrations}

Tegafur, CDHP, and 5-fluorouracil concentrations in tears and plasma were determined by the method described by Liu et al. [8], with minor modifications. The methods are described in detail in the Supplementary Methods.

\section{Statistical considerations and sample size}

To calculate the initial sample size, the incidence of LDO was presumed to be about $20 \%$ according to our previous study [2]. A total of 200 patients were required to detect an 


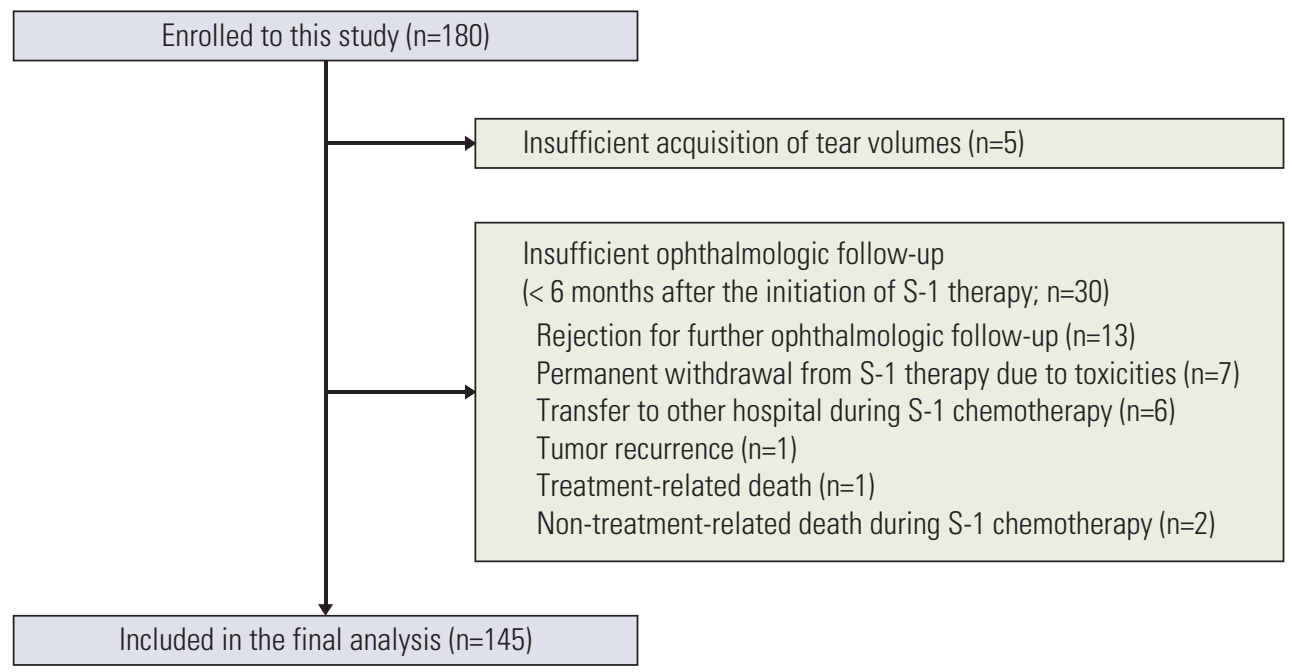

Fig. 1. CONSORT diagram of the patients in this study.

effect size of 0.5 in the 5 -fluorouracil concentration, assuming a power of $80 \%$ at a significance level of 0.05 . During the early phase in this study, we observed that the actual incidence of LDO was likely to be higher than expected. Therefore, in April 2012, we recalculated the sample size as 140 (49 patients with LDO and 91 without LDO) based on the revised assumption of the incidence of $\mathrm{LDO}$ (about $35 \%$ ). We aimed to recruit 180 patients considering the possibility of early dropouts (about 25\%) secondary to toxicity or a failure to return for a scheduled ophthalmologic follow-up visit. The study protocol recommended that the ophthalmologic evaluation be conducted until the completion of S-1 therapy if LDO did not develop during S-1 therapy. However, since the cumulative incidence of LDO reached a plateau around 6 months after the initiation of S-1 in our previous study [2], the protocol considered patients without the LDO development to have completed the required ophthalmologic followup visits if ophthalmologic evaluation was conducted until at least 6 months after the initiation of S-1. As planned, patients without LDO development, whose ophthalmologic follow-up was less than six months, were excluded from the final analyses.

The cumulative incidence of LDO was calculated from the date of S-1 initiation until the date of LDO diagnosis using the Kaplan-Meier method. Log-rank tests were conducted to investigate differences in the incidence of LDO among comparison groups in univariable analyses. Cox proportional hazards models were conducted to investigate the impact of specific risk factors on LDO development in multivariable analysis. IBM SPSS for Windows (IBM Corp., Armonk, NY) was used for data analysis, and p-values of $<0.05$ were considered significant. All tests were two-tailed.

\section{Results}

\section{Patient characteristics}

A total of 180 patients were initially enrolled in this study. Of these, five were excluded owing to inadequate tear volume acquisition. Thirty patients who did not develop LDO and whose ophthalmologic follow-up period was less than six months were also excluded (Fig. 1). Therefore, 145 patients were included in the final analysis. The median age was 59 years (range, 22 to 82 years) and the median level of creatinine clearance rate (Ccr) was $79 \mathrm{~mL} / \mathrm{min}$ (range, 41 to $148 \mathrm{~mL} /$ min). Among the 145 patients, 137 (94\%) completed the planned 1-year S-1 therapy. Detailed patient characteristics are presented in Table 1.

\section{Development of LDO}

Fifty-three patients $(37 \%, 53 / 145)$ developed LDO, and the cumulative incidences of LDO were $22 \%, 31 \%, 36 \%$, and $37 \%$ at 3, 6, 9, and 12 months after the S-1 initiation, respectively (Fig. 2). Among the 53 patients with LDO, 45 underwent dacryocystography, and all the cases showed narrowing or obstruction of the lacrimal drainage system. The median time 
Table 1. Patient characteristics

\begin{tabular}{|c|c|}
\hline Clinical characteristic & No. $(\%)$ \\
\hline Age, median (range, yr) & $59(22-82)$ \\
\hline \multicolumn{2}{|l|}{ Sex } \\
\hline Male & $100(69)$ \\
\hline Female & $45(31)$ \\
\hline \multicolumn{2}{|l|}{ Stage } \\
\hline IB & $2(1)$ \\
\hline II & $92(63)$ \\
\hline III & $51(35)$ \\
\hline \multicolumn{2}{|l|}{ Pathology } \\
\hline $\begin{array}{l}\text { Well or moderately differentiated } \\
\text { adenocarcinoma }\end{array}$ & $60(41)$ \\
\hline Poorly differentiated adenocarcinoma & $30(21)$ \\
\hline Signet ring cell carcinoma & $45(31)$ \\
\hline Others & $10(7)$ \\
\hline \multicolumn{2}{|l|}{ Lauren classification } \\
\hline Intestinal type & $62(43)$ \\
\hline Diffuse type & $72(50)$ \\
\hline Mixed or intermediate type & $11(8)$ \\
\hline \multicolumn{2}{|l|}{ Primary tumor location } \\
\hline Lower third & $73(50)$ \\
\hline Middle third & $21(14)$ \\
\hline Upper third & $31(21)$ \\
\hline$\geq 2 / 3$ of stomach & $20(14)$ \\
\hline \multicolumn{2}{|l|}{ Gross morphology of primary tumor } \\
\hline Early gastric cancer & $21(14)$ \\
\hline Borrmann type I & $4(3)$ \\
\hline Borrmann type II & $19(13)$ \\
\hline Borrmann type III & $91(63)$ \\
\hline Borrmann type IV & $10(7)$ \\
\hline \multicolumn{2}{|l|}{ Method of gastrectomy } \\
\hline Laparoscopic surgery & $114(79)$ \\
\hline Open surgery & $31(21)$ \\
\hline \multicolumn{2}{|l|}{ Extent of gastrectomy } \\
\hline Total gastrectomy & $41(28)$ \\
\hline Distal gastrectomy & $99(68)$ \\
\hline Proximal gastrectomy & $5(3)$ \\
\hline Creatinine clearance, median (range, $\mathrm{mL} / \mathrm{min}$ ) & $79(41-148)$ \\
\hline
\end{tabular}

Adjuvant chemotherapy was conducted in patients with pathologic stages II-III according to the American Joint Committee on Cancer (AJCC, 7th edition). However, two patients who had stage IB with additional risk factors (N2 lymph node metastasis according to the Japanese staging classification) were also included.

to the development of LDO in these 53 patients was 10.9 weeks (95\% confidence interval, 9.4 to 12.4; range, 1.0 to 43.9 weeks) after the initiation of S-1, and $85 \%$ of the LDO cases $(45 / 53)$

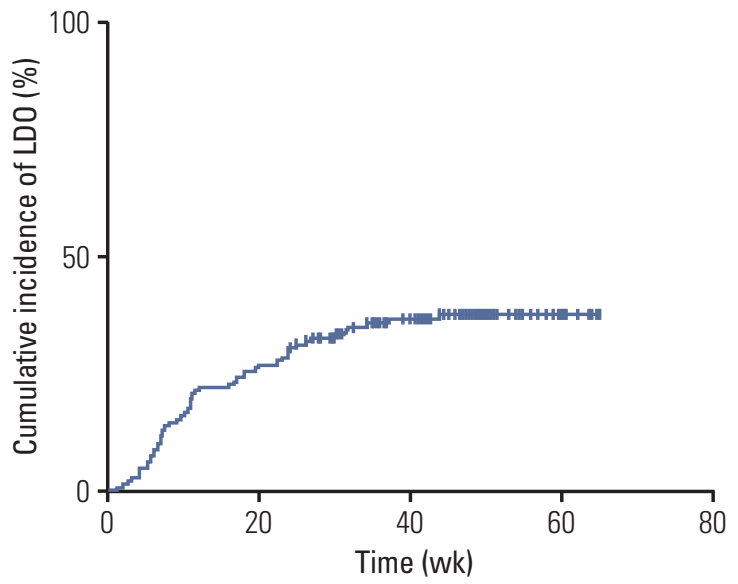

Fig. 2. A Kaplan-Meier curve of the cumulative incidence of lacrimal drainage obstruction (LDO) in patients with gastric cancer receiving adjuvant S-1 chemotherapy $(\mathrm{n}=145)$. Fifty-three patients $(37 \%)$ developed LDO, and the cumulative incidences of LDO were $22 \%, 31 \%, 36 \%$, and $37 \%$ at 3, 6, 9, and 12 months after the initiation of S-1 therapy, respectively.

were developed within 6 months of starting the S-1 treatment. The most common site of obstruction was the nasolacrimal duct $(n=42)$, followed by the punctum $(n=15)$ and canaliculus $(n=13)$ (S1 Fig.). Fifteen patients had LDO at two or more levels simultaneously. When additional analysis was conducted that included all patients initially enrolled into this study $(n=180)$, the cumulative incidence of LDO was not different from that for the 145 patients included in the final analysis (S2 Fig.). Management and clinical course of LDO are described in detail in the supplementary file (S3 Fig.).

\section{Concentrations of tegafur, 5-fluorouracil, and CDHP in tears and plasma}

We measured the trough concentrations of tegafur, CDHP, and 5-fluorouracil in a steady state in both tears and plasma. However, only tegafur was detected in tears, while neither CDHP nor 5-fluorouracil was detected. All three components were detected in plasma. Correlations among the concentrations of tear tegafur, plasma tegafur, plasma 5-fluorouracil, plasma CDHP, and the level of Ccr were explored. We found strong positive correlations among the tear tegafur, plasma tegafur, plasma 5-fluorouracil, and plasma CDHP concentrations. In contrast, negative correlations were observed among Ccr and the plasma 5-fluorouracil and plasma CDHP concentrations (Fig. 3). 


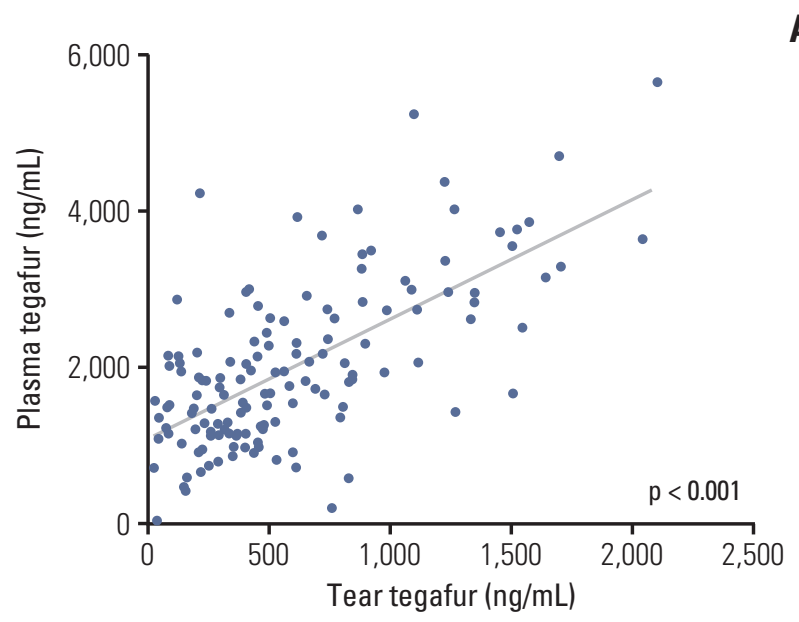

A

B
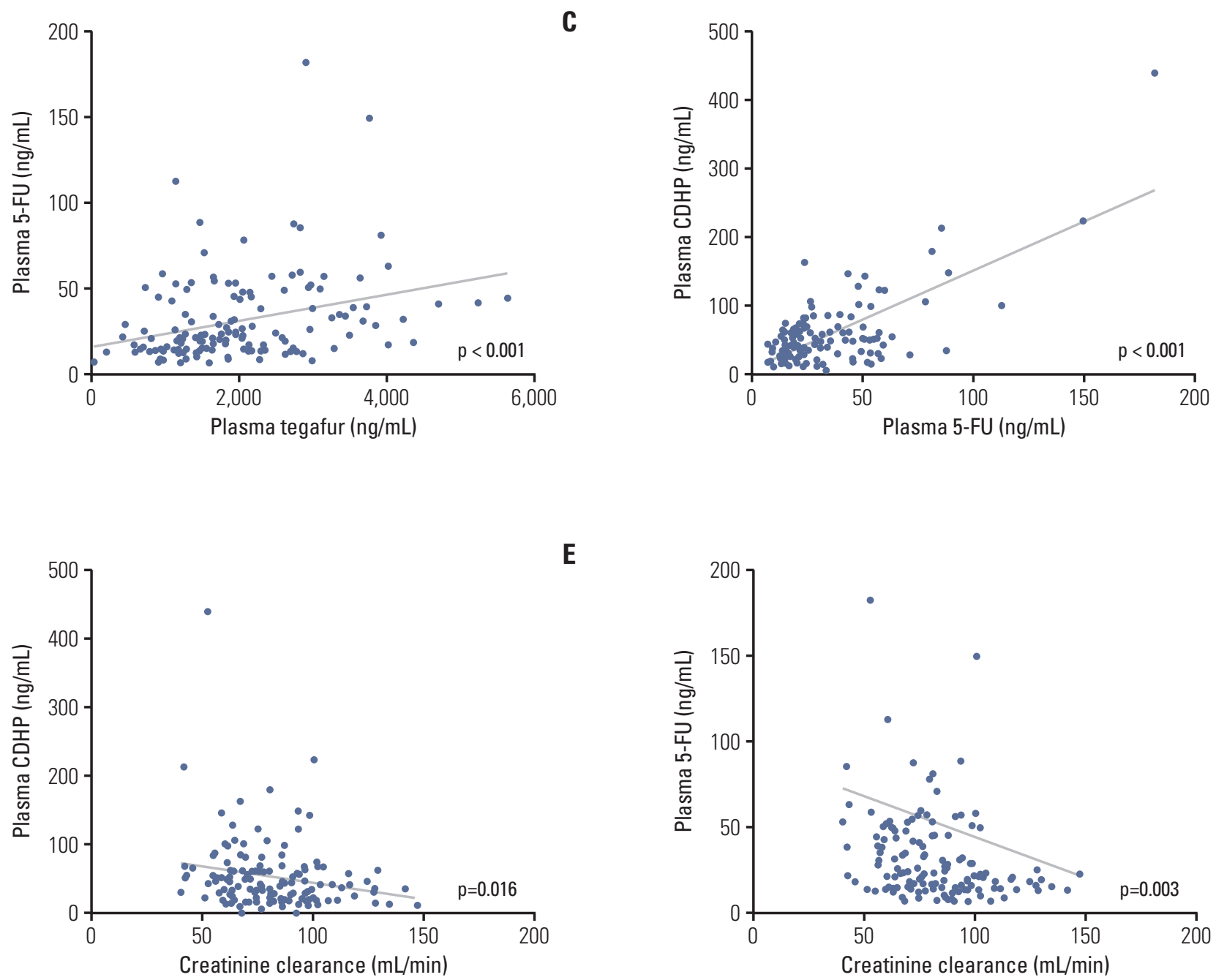

$\mathbf{E}$

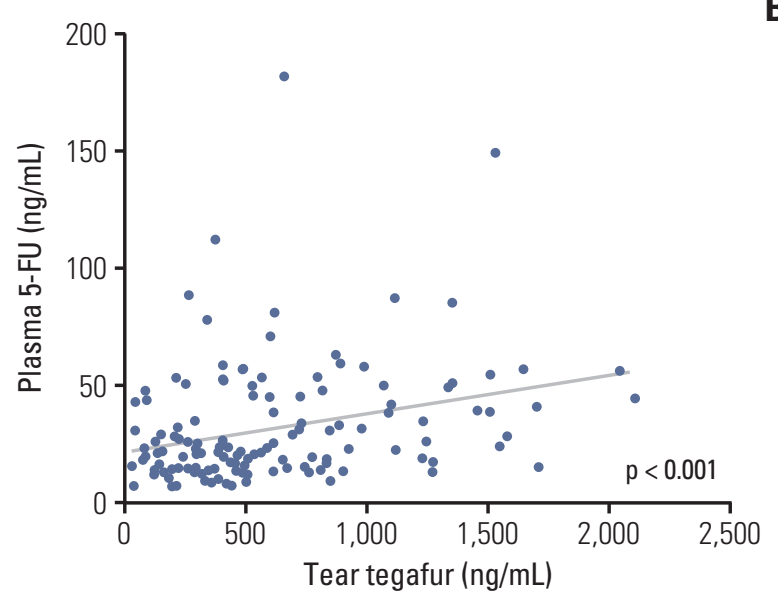

C

D

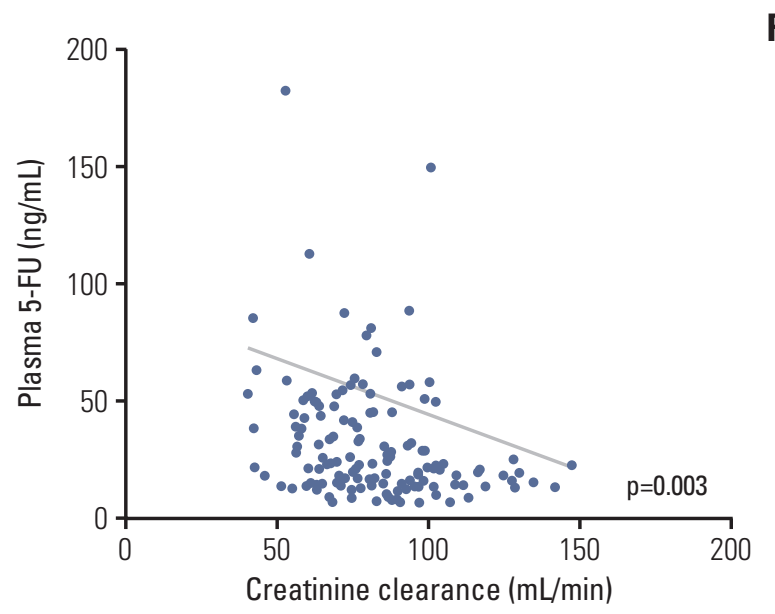

Fig. 3. (A-F) Correlations among the concentrations of tear tegafur, plasma tegafur, plasma 5-fluorouracil (5-FU), plasma 5-chloro-2,4-dihydroxypyridine (CDHP), and the creatinine clearance rate. 
Table 2. Univariable and multivariable analyses of risk factors for the development of lacrimal drainage obstruction

\begin{tabular}{|c|c|c|c|c|c|}
\hline \multirow[b]{2}{*}{ Characteristic } & \multicolumn{2}{|c|}{ Univariable analysis ${ }^{a)}$} & \multicolumn{3}{|c|}{ Multivariable analysis ${ }^{\text {a) }}$} \\
\hline & $\begin{array}{l}\text { 1-Year cumulative } \\
\text { incidence of } \\
\text { LDO }(\%)\end{array}$ & p-value & $\begin{array}{l}\text { Hazard } \\
\text { ratio }\end{array}$ & $\begin{array}{l}95 \% \text { Confidence } \\
\text { interval }\end{array}$ & p-value \\
\hline \multicolumn{6}{|l|}{ Sex } \\
\hline Male & 41.4 & 0.207 & 1.00 & - & - \\
\hline Female & 29.8 & & 0.88 & $0.45-1.70$ & 0.697 \\
\hline \multicolumn{6}{|l|}{ Age (yr) } \\
\hline$<70$ & 30.4 & $<0.001$ & 1.00 & - & - \\
\hline$\geq 70$ & 68.4 & & 1.99 & $1.00-3.95$ & 0.049 \\
\hline \multicolumn{6}{|l|}{ Extent of gastrectomy } \\
\hline Partial gastrectomy & 34.6 & 0.201 & 1.00 & - & - \\
\hline Total gastrectomy & 46.0 & & 1.17 & $0.60-2.29$ & 0.641 \\
\hline Creatinine clearance $(\mathrm{Ccr}, \mathrm{mL} / \mathrm{min})$ & & & & & 0.018 \\
\hline$\geq 80$ & 20.0 & $<0.001$ & 1.00 & - & - \\
\hline$\geq 60$ and $<80$ & 52.3 & & 2.52 & $1.21-5.22$ & 0.013 \\
\hline$<60$ & 63.7 & & 3.21 & $1.35-7.65$ & 0.008 \\
\hline \multicolumn{6}{|l|}{$\begin{array}{l}\text { Plasma 5-fluorouracil concentration } \\
\text { (median, } 22.3 \mathrm{ng} / \mathrm{mL})^{\text {b) }}\end{array}$} \\
\hline$<22.3$ & 22.8 & $<0.001$ & 1.00 & - & - \\
\hline$\geq 22.3$ & 52.1 & & 2.02 & $1.03-3.93$ & 0.040 \\
\hline \multicolumn{6}{|l|}{ 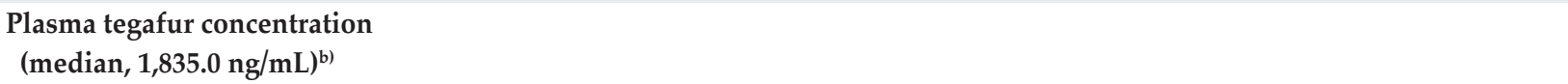 } \\
\hline$<1,835.0$ & 34.4 & 0.528 & 1.00 & - & - \\
\hline$\geq 1,835.0$ & 40.5 & & 0.54 & $0.29-1.03$ & 0.062 \\
\hline \multicolumn{6}{|l|}{$\begin{array}{l}\text { Plasma CDHP concentration } \\
(\text { median, } 42.0 \mathrm{ng} / \mathrm{mL})^{\text {b) }}\end{array}$} \\
\hline$<42.0$ & 30.4 & 0.032 & 1.00 & - & - \\
\hline$\geq 42.0$ & 45.3 & & 1.22 & $0.63-2.37$ & 0.559 \\
\hline \multicolumn{6}{|l|}{$\begin{array}{l}\text { Tear tegafur concentration } \\
(\text { median, } 479.2 \mathrm{ng} / \mathrm{mL})^{\text {b) }}\end{array}$} \\
\hline$<479.2$ & 26.7 & 0.006 & 1.00 & - & - \\
\hline$\geq 479.2$ & 50.0 & & 1.85 & $0.99-3.45$ & 0.055 \\
\hline
\end{tabular}

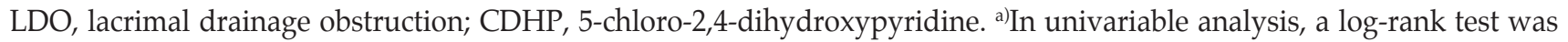

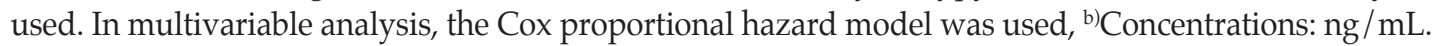

\section{Risk factors for the development of LDO}

In univariable analyses conducted to identify predictors of the LDO development, clinical parameters and the S-1 component (or 5-fluorouracil) concentrations in both tears and plasma were included. Among the clinical parameters, the older age ( $\geq 70$ years) and decreased $\mathrm{Ccr}(<80 \mathrm{~mL} / \mathrm{min})$ were related to the increased LDO development $(\mathrm{p}<0.05)$. The sex or extent of gastrectomy was not predictive of LDO development. Regarding the S-1-related compounds, patients with increased concentrations of plasma 5-fluorouracil $(\geq 22.3$ $\mathrm{ng} / \mathrm{mL}$ [median], $\mathrm{p}<0.001)$, plasma CDHP $(\geq 42.0 \mathrm{ng} / \mathrm{mL}$ [median], $\mathrm{p}=0.032)$, and tear tegafur $(\geq 479.2 \mathrm{ng} / \mathrm{mL}$ [median], $\mathrm{p}=0.006$ ) developed LDO more frequently. However, the concentration of plasma tegafur was not associated with LDO development (Table 2, Fig. 4).

Multivariable analysis revealed that older age and decreased Ccr were independent risk factors for LDO development. Specifically, as the level of Ccr decreased, the risk of LDO increased, as indicated by a hazard ratio (HR) of 2.52 in patients with $60 \leq \mathrm{Ccr}<80$ and 3.21 in those with $\mathrm{Ccr}<60$ $\mathrm{mL} / \mathrm{min}$. Among the S-1-related compounds, only a high concentration of plasma 5-fluorouracil was predictive of the increased LDO development $(\mathrm{HR}, 2.02 ; \mathrm{p}=0.040)$. The patients 

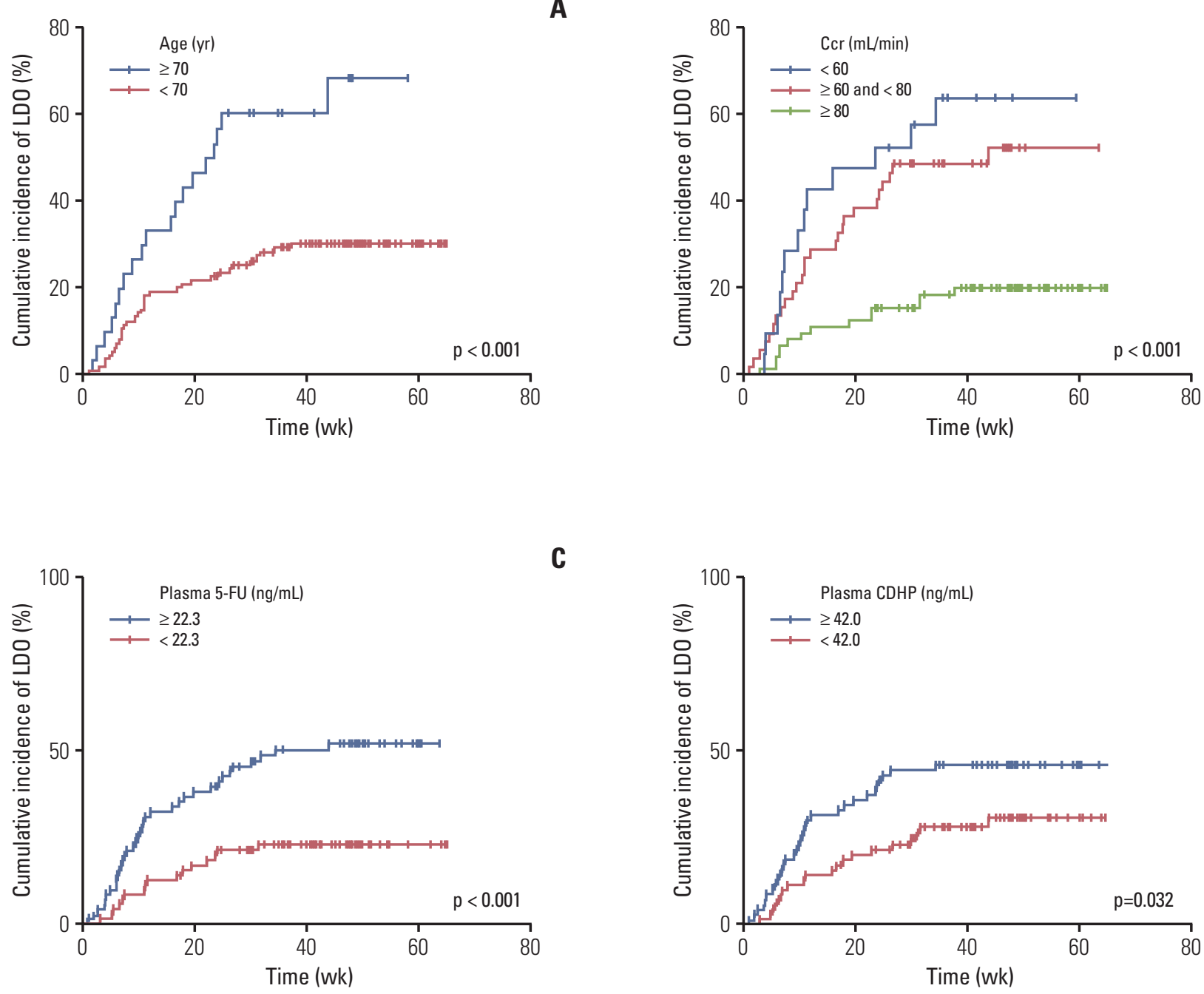

C

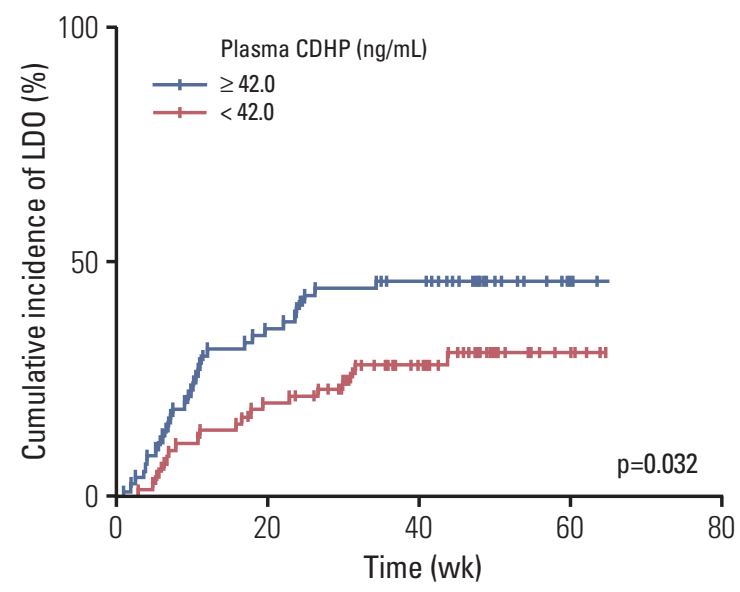

Fig. 4. Univariable analysis of risk factors for the development of lacrimal drainage obstruction (LDO). (A) Patients aged $\geq 70$ years developed LDO more commonly than those aged $<70$ years (long-rank test, $p<0.001$ ). (B) Three groups of patients with different levels of creatinine clearance (Ccr $\geq 80 \mathrm{~mL} / \mathrm{min}, 60 \mathrm{~mL} / \mathrm{min} \leq \mathrm{Ccr}<80 \mathrm{~mL} / \mathrm{min}$, and $\mathrm{Ccr}<60 \mathrm{~mL} / \mathrm{min}$ ) showed different incidences of LDO, exhibiting an increased incidence of LDO development with decreased Ccr (log-rank test, $\mathrm{p}<0.001$ ). (C) Patients with high 5-fluorouracil (5-FU) concentrations in plasma ( $\geq 22.3 \mathrm{ng} / \mathrm{mL}$ [median]) developed LDO more frequently than those with low plasma 5-FU concentrations $(\mathrm{p}<0.001)$. (D) Patients with high 5-chloro-2,4-dihydroxypyridine (CDHP) concentrations in plasma ( $\geq 42.0 \mathrm{ng} / \mathrm{mL}$ [median]) developed LDO more frequently than those with low plasma CDHP concentrations ( $\mathrm{p}=0.032$ ). (Continued to the next page)

with a high tear tegafur concentration tended to show increased LDO development, but the trend was not statistically significant $(\mathrm{p}=0.055)$. The concentrations of plasma tegafur or CDHP did not show an independent effect on LDO development in the multivariable model (Table 2).

\section{LDO and other S-1 toxicities}

The toxicity profiles developed during S-1 therapy are presented in the supplementary file (S4 Table). Frequencies of hematologic and non-hematologic toxicities were compared between patients with and without LDO. Patients who developed LDO also developed non-hematologic toxicities (grade $\geq 2$ ) more frequently than those without LDO ( $p=0.016)$. In 


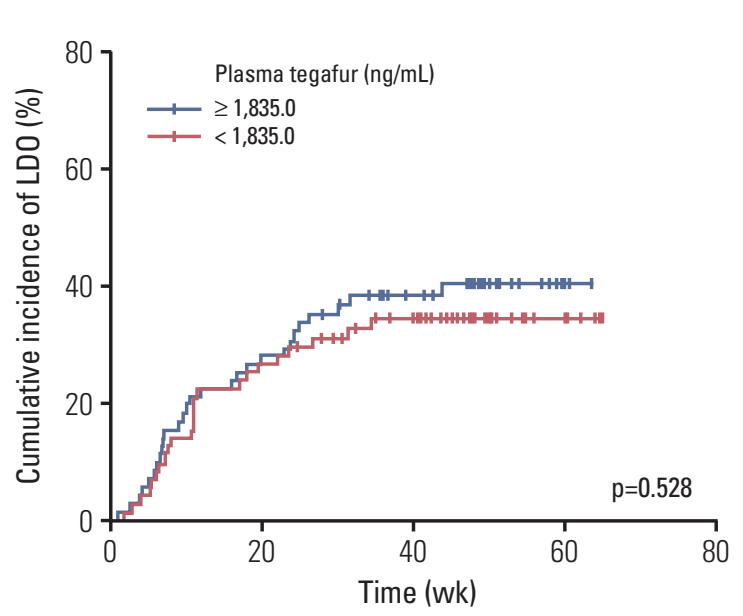

E

Fig. 4. (Continued from the previous page) (E) There was no difference in the development of LDO between patients with high and low plasma tegafur concentrations (median, $1,835.0 \mathrm{ng} / \mathrm{mL} ; \mathrm{p}=0.528$ ). (F) Patients with high tegafur concentrations in tears $(\geq 479.2 \mathrm{ng} / \mathrm{mL}$ [median]) developed LDO more frequently than those with low tear tegafur concentrations $(\mathrm{p}=0.006)$.

Table 3. Comparison of the incidences of S-1-related hematologic and non-hematologic toxicities in patients with and without LDO

\begin{tabular}{lccc} 
& Patients without LDO (n=92) & Patients with LDO (n=53) & p-value ${ }^{\mathrm{a})}$ \\
Hematologic toxicity & & & \\
$\quad$ Grade 0 or 1 & $52(56.5)$ & $22(58.5)$ & 0.817 \\
$\quad \geq$ Grade 2 & $40(43.5)$ & & \\
Non-hematologic toxicity & & $11(20.8)$ & 0.016 \\
Grade 0 or 1 & $37(40.2)$ & $42(79.2)$ & \\
$\geq$ Grade 2 & $55(59.8)$ & & \\
\hline
\end{tabular}

Values are presented as number (\%). LDO, lacrimal drainage obstruction. ${ }^{\mathrm{a}}$ Chi-square test.

contrast, there was no difference in the frequencies of hematologic toxicities between patients with and without LDO (Table 3).

\section{Discussion}

In this prospective study, we found that the incidence of LDO in patients receiving S- 1 chemotherapy was 37\%, which is much higher than the incidence $(8 \%-18 \%)$ reported in previous retrospective studies [2-5]. Some patients would not seek medical care; therefore, the incidence of S-1-induced LDO is thought to have been underestimated in these studies. We also found that the development of S-1-induced LDO is related to an older age, decreased Ccr, and an increased plasma concentration of 5-fluorouracil.

There have been a few studies in which tear concentrations of systemically administered drugs, such as docetaxel and 5-fluorouracil [9-11], were measured. Esmaeli et al. [9] reported that docetaxel was found in tear samples collected immediately after docetaxel infusion and suggested that the secretion of docetaxel in tears might be a mechanism for tear drainage obstruction. Loprinzi et al. [11] reported that 5-fluorouracil was detectable in the tears of 12 patients within several minutes after the 5 -fluorouracil infusion; however, they stated that the tear concentrations of 5-fluorouracil were not correlated with the development of ocular toxicity. Our study is the first prospective report on the incidence of S-1-induced LDO and the only report that analyzed the concentrations of S-1 components in tears. We collected tears 
and plasma when the drug concentration was at a steadystate trough level to minimize the interpatient variations in S-1 component (or 5-fluorouracil) concentrations under the same conditions. The concentration of CDHP or 5-fluorouracil could not be measured, and only the tegafur concentration could be analyzed in tears. Considering that the median CDHP/tegafur and 5-fluorouracil/tegafur ratios in plasma were 0.02 and 0.01 , respectively, and the tear-toplasma ratio of the median tegafur concentrations was 0.26 , the concentrations of CDHP and 5-fluorouracil in tears are thought to be in a too low range to be detected by the analysis system.

We demonstrated that the plasma concentration of 5-fluorouracil is an independent risk factor for LDO development. Non-hematologic toxicities were more frequent in patients who developed LDO. These findings strongly suggest that the S-1-induced LDO is caused by damage to and inflammation of the lacrimal drainage system, mainly due to the systemic effects of S-1 rather than to direct contact between the mucosal lining of the lacrimal drainage system and S-1 components or 5-fluorouracil in tears [12]. This suggested mechanism also explains the finding that S-1-induced LDO is more frequently located in the nasolacrimal duct (distal lacrimal drainage) than in puncta or canaliculus (proximal lacrimal drainage). In fact, there are abundant vascular plexuses in the wall of the nasolacrimal duct; therefore, the systemic blood supply easily reaches the submucosal space of the nasolacrimal duct $[13,14]$. If the local effect of S-1 components or 5-fluorouracil on the development of LDO was predominant, obstructions in the puncta or canaliculus would be more common than those in the nasolacrimal duct. The frequent development of LDO in older patients or in patients with decreased Ccr would also be explained by a systemic effect of S-1.

However, we cannot completely exclude the possibility that S-1 components or 5-fluorouracil in tears exert a local effect on the lacrimal drainage system and additionally contribute to the S-1-induced LDO development since tegafur was only detected in tears with our analysis equipment. The high tegafur concentration in tears was related to the increased LDO development upon univariable analysis, but this statistical significance disappeared $(\mathrm{p}=0.055)$ when the multivariable analysis was conducted. The concentrations of $\mathrm{CDHP}$ and 5-fluorouracil in tears were too low to be detected by the analysis equipment; thus, we think that their local effects on the LDO development would be minor, if any. In addition, we measured the concentrations of the S-1-related compounds at the steady-state trough level, but not at the steady-state peak level. Thus, we cannot completely exclude the possibility that the development of S-1-induced LDO might be more influenced by peak levels of S-1 ingredients / metabolites when compared to the effects caused by the trough levels.

In our previous retrospective study [2], we reported that total gastrectomy was a risk factor for the development of S1-induced LDO. However, total gastrectomy did not seem to be related to the increased development of LDO in the present study (Table 2, S5 Fig.), although there was a considerable difference in the 1-year cumulative incidence of LDO between total gastrectomy $(46.0 \%)$ and partial gastrectomy $(34.6 \%)$. Since the sample size in the present study aimed to provide sufficient power for detecting differences in 5-fluorouracil concentration, but not for detecting differences in LDO incidence, further studies are needed to verify whether total gastrectomy is a risk factor of S-1-induced LDO.

Regarding cutoff values of tear or plasma concentrations of S-1 ingredients/metabolites, we used a median value of each component because there were no references that could guide us to determine the most appropriate cutoff values. Because we wanted to exclude an unexpected bias derived from arbitrary analyses to determine the cutoff values, we selected the median concentration value of each component as the cutoff. When we separated patients into three groups using different cutoff values with tertiles and conducted univariable analyses, the cutoff values of plasma 5 -fluorouracil, plasma CDHP and tear tegafur concentrations also classified patients well into two groups with high or low risks of developing LDO (S6 Fig.). Therefore, the results from these univariable analyses show that, although the most appropriate cutoff points are uncertain, the development of LDO is really affected by the concentrations of S- 1 ingredients / metabolites.

In conclusion, our study shows that LDO is a frequent adverse event in patients receiving S-1 chemotherapy and strongly suggests that it is caused by one of the S-1-induced systemic adverse effects rather than by local effects of S-1. Clinical risk factors (older age and decreased renal function) were also identified. Oncologists must be alert to eye symptoms in patients receiving S-1 treatment and cooperate with ophthalmologists to improve the quality of life of patients receiving S-1 therapy. Our study also warrants further investigations of prevention of LDO development or on the treatment strategy to intervene with LDO at an early stage before more complicated surgical procedures are required in the late stage of LDO.

\section{Electronic Supplementary Material}

Supplementary materials are available at Cancer Research and Treatment website (http:// www.e-crt.org).

\section{Conflicts of Interest}

Conflict of interest relevant to this article was not reported. 


\section{Acknowledgments}

The study was partly supported by a research grant (03-2013-003) from the Seoul National University Bundang Hospital.

\section{Author Details}

Departments of ${ }^{1}$ Ophthalmology and ${ }^{2}$ Internal Medicine, Seoul National University Bundang Hospital, Seoul National University College of Medicine, Seongnam, ${ }^{3}$ Center of Biomedical Mass Spec- trometry, Diatech Korea Co., Ltd., Seoul, Departments of ${ }^{4}$ Internal Medicine and ${ }^{5} \mathrm{Ophthalmology}$, Seoul National University Boramae Medical Center, Seoul, Departments of ${ }^{6}$ Internal Medicine and ${ }^{7}$ Ophthalmology, Seoul National University College of Medicine, Seoul National University Hospital, Seoul, Departments of ${ }^{8}$ Surgery and ${ }^{9}$ Clinical Pharmacology and Therapeutics, Seoul National University Bundang Hospital, Seoul National University College of Medicine, Seongnam, ${ }^{10}$ Medical Research Collaborating Center, Seoul National University Bundang Hospital, Seongnam, Korea

\section{References}

1. Satoh T, Sakata Y. S-1 for the treatment of gastrointestinal cancer. Expert Opin Pharmacother. 2012;13:1943-59.

2. Kim N, Park C, Park DJ, Kim HH, Kim S, Kim YJ, et al. Lacrimal drainage obstruction in gastric cancer patients receiving S-1 chemotherapy. Ann Oncol. 2012;23:2065-71.

3. Kitamura H, Miyanaga T, Shin H, Fujita M, Miyazaki M, Yagi D, et al. Investigation of epiphora following S-1 therapy. Gan To Kagaku Ryoho. 2011;38:259-62.

4. Sasaki T, Miyashita H, Miyanaga T, Yamamoto K, Sugiyama K. Dacryoendoscopic observation and incidence of canalicular obstruction/stenosis associated with S-1, an oral anticancer drug. Jpn J Ophthalmol. 2012;56:214-8.

5. Sato K, Nishimura S. Adverse effects of the oral anticancer drug s-1: lacrimal passage impairment and specific features of corneal epitheliopathy. Open Ophthalmol J. 2013;7:85-6.

6. Kim SJ, Kim YJ, Kim JH, Park DJ, Kim HH, Lee JS, et al. Safety, compliance, and predictive parameters for dosage modification in adjuvant S-1 chemotherapy for gastric cancer. Cancer Sci. 2013;104:116-23.

7. Kim JY, Shin E, Kim JW, Lee HS, Lee DW, Kim SH, et al. Impact of intratumoral expression levels of fluoropyrimidinemetabolizing enzymes on treatment outcomes of adjuvant S-1 therapy in gastric cancer. PLoS One. 2015;10:e0120324.

8. Liu K, Zhong D, Zou H, Chen X. Determination of tegafur, 5-fluorouracil, gimeracil and oxonic acid in human plasma using liquid chromatography-tandem mass spectrometry. J Pharm Biomed Anal. 2010;52:550-6.

9. Esmaeli B, Ahmadi MA, Rivera E, Valero V, Hutto T, Jackson $\mathrm{DM}$, et al. Docetaxel secretion in tears: association with lacrimal drainage obstruction. Arch Ophthalmol. 2002;120: 1180-2.

10. Christophidis N, Vajda FJ, Lucas I, Louis WJ. Ocular side effects with 5-fluorouracil. Aust N Z J Med. 1979;9:143-4.

11. Loprinzi CL, Love RR, Garrity JA, Ames MM. Cyclophosphamide, methotrexate, and 5-fluorouracil (CMF)-induced ocular toxicity. Cancer Invest. 1990;8:459-65.

12. Esmaeli B, Valero V, Ahmadi MA, Booser D. Canalicular stenosis secondary to docetaxel (taxotere): a newly recognized side effect. Ophthalmology. 2001;108:994-5.

13. Paulsen FP, Thale AB, Hallmann UJ, Schaudig U, Tillmann BN. The cavernous body of the human efferent tear ducts: function in tear outflow mechanism. Invest Ophthalmol Vis Sci. 2000;41:965-70.

14. Thale A, Paulsen F, Rochels R, Tillmann B. Functional anatomy of the human efferent tear ducts: a new theory of tear outflow mechanism. Graefes Arch Clin Exp Ophthalmol. 1998;236:674-8. 